\title{
Successful treatment of exogenous aspergillus endophthalmitis: a case report
}

\author{
PATRICK C. HO, ' FELIPE I. TOLENTINO, ${ }^{2}$ AND ANN S. BAKER ${ }^{3}$ \\ From the ${ }^{1-3}$ Massachusetts Eye and Ear Infirmary and ${ }^{12}$ the Eye Research Institute of Retina Foundation, \\ Boston, USA
}

SUMMARY We describe the first case of successfully treated exogenous aspergillus endophthalmitis following penetrating injury and primary scleral wound repair. After repeated vitreous surgery with multiple intravitreous instillations of amphotericin B combined with intravenous antifungal therapy the patient's visual acuity was 6/18. A vigorous approach in the management of mycotic endophthalmitis is emphasised and the role of closed vitrectomy discussed.

Aspergillus appears occasionally in keratitis and orbital infection and infrequently has been reported as a cause of endophthalmitis. Intraocular aspergillus infection carries a grave prognosis. Of the 38 cases of aspergillus endophthalmitis reported to date, of which 24 were endogenous ${ }^{1-3}$ and 14 exogenous, ${ }^{4}$ useful vision was saved in only 2 endogenous cases.

We describe a case of endophthalmitis caused by Aspergillus fumigatus that followed penetrating injury and primary repair of a scleral wound. Vitrectomy combined with intravitreous and intravenous amphotericin B therapy resulted in the preservation of useful vision. To our knowledge this is the first case of exogenous aspergillus endophthalmitis with a good visual result.

\section{Case report}

A 28-year-old man was hit in the right eye by a metal band that broke from a bale while he was unloading a truck. He was in perfect health and had no history of intravenous drug abuse.

When we examined him on the afternoon of the accident visual acuity in the right eye was counting fingers at 4 feet $(1.2 \mathrm{~m})$ and in the left eye $6 / 6$. The right eye was mushy soft with a clear cornea. Marked fibrinoid reaction occupied a shallow anterior chamber. The lens was clear behind a mid-dilated pupil. An anterior scleral laceration was easily detected in the superonasal quadrant. A red fundus reflex was present. Immediately $2 \mathrm{~g}$ cephalothin was

Correspondence to the Library, Eye Research Institute, 20 Staniford Street, Boston, MA 02114, USA. given intravenously together with $0.5 \mathrm{ml}$ of intramuscular tetanus toxoid. After radiography ruled out the presence of a metallic intraocular foreign body the patient was operated on.

After a specimen had been taken for culture of the scleral wound the vitreous gel prolapsing through the wound was excised. The scleral wound, which measured $9 \mathrm{~mm}$ in length and extended from the 1 o'clock meridian, where it was $5 \mathrm{~mm}$ from the limbus, to the 3 o'clock meridian, where it was $1 \mathrm{~mm}$ from the limbus, was repaired with 8-0 nylon suture. After the intraocular pressure was reformed, with injection of balanced salt solution, the repaired scleral wound was treated with cryoapplication. Indirect ophthalmoscopy showed that the retina was attached at the end of the operation. A postoperative wound culture was taken.

The patient recovered uneventfully from surgery. He received a 5-day course of intravenous cephalothin $(4 \mathrm{~g} /$ day $)$ and gentamicin $(240 \mathrm{mg} /$ day $)$ in divided doses. Topical gentamicin, prednisolone acetate, and atropine were also given. The wound cultures yielded no growth, and the patient was discharged on the sixth postoperative day with clear media and an attached retina.

When he returned 10 days later, vision in the right eye was 6/9. Through clear media tortuosity of the retinal blood vessels and small retinal haemorrhages scattered in the periphery were detected (Fig. 1). Late that evening the patient experienced a precipitous onset of ocular pain and rapid loss of vision in the right eye. When he was examined the following morning, visual acuity in the right eye had dropped to hand movements at 2 feet $(60 \mathrm{~cm})$. The upper and 


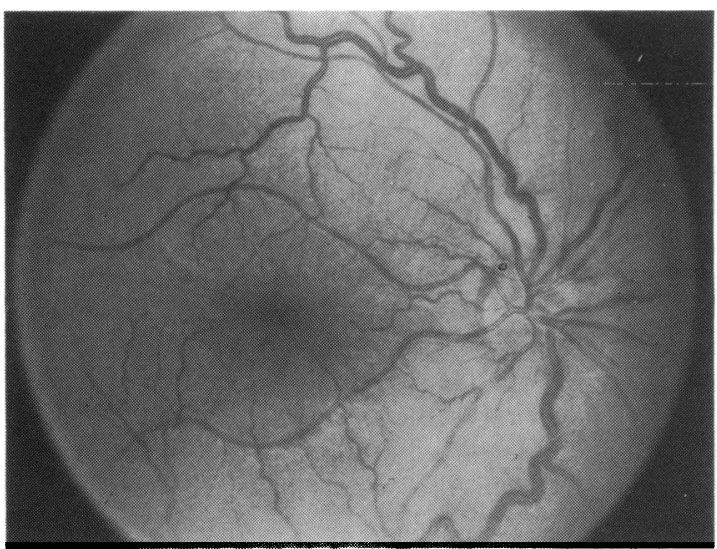

Fig. 1 Fundus photograph of the right eye 16 days after the injury and scleral wound repair. Visual acuity was 6/9. The patient experienced sudden onset of severe ocular pain and decrease of vision the evening the photograph was taken.

lower lids were swollen, and the conjunctiva was markedly injected. Numerous cells and a great deal of flare in the anterior chamber and vitreous cavity prevented our seeing any fundus details. The intraocular pressure was $10 \mathrm{mmHg}$.

Because of the possibility of endophthalmitis we took the patient to the operating room immediately. After a preoperative specimen had been taken from the wound for culture, vitreous fluid was aspirated from a pars plana sclerotomy and sent for culture. A closed vitrectomy was then performed, and the fluid removed during the vitrectomy was cultured. The operation was completed with intravitreous injection of cephazolin $(1 \mathrm{mg})$ and gentamicin $(400 \mu \mathrm{g})$. Intravenous cephalothin $(2 \mathrm{~g})$ and hydrocortisone $(100 \mathrm{mg})$ were also given together with subconjunctival injection of gentamicin $(40 \mathrm{mg})$ and cephalothin (100 mg).

Postoperatively the fundus view was not restored, though the anterior chamber was clear and the patient was free of pain. Before the culture results were available he was given subconjunctival gentamicin $(40 \mathrm{mg})$ and cephalothin $(100 \mathrm{mg})$ and intravenous gentamicin (80 mg every 8 hours) and cephalothin (2 g every 6 hours). Topically he received gentamicin and dexamethasone phosphate every 2 hours and atropine (1\%) 4 times a day.

Fortyeight hours after vitrectomy the wound culture yielded no growth, whereas Aspergillus fumigatus was recovered from the cultures of the vitreous aspirate and the vitreous fluid collected during vitrectomy. The patient was taken to the operating room, and under local anaesthesia $7.5 \mu \mathrm{g}$ of amphotericin B was injected slowly into the anterior vitreous after vitreous fluid was aspirated for repeat culture. (Aspergillus fumigatus was isolated from the repeat vitreous culture.) Intravenous amphotericin B, $1 \mathrm{mg}$ test dose then $25 \mathrm{mg}$, was begun.

The next day a red fundus reflex could be observed. Intraocular pressure was $12 \mathrm{mmHg}$. Oral prednisone $(80 \mathrm{mg} /$ day) was given. One day later intraocular pressure fell to $4 \mathrm{mmHg}$. The anterior chamber contained a moderate amount of cells and flare. The lens became hazy with a white retrolental mass. The red fundus reflex could no longer be detected. That evening under general anaesthesia the patient underwent lensectomy and vitreous washout in the right eye. Retrolental and cyclitic membranes were also excised, $5 \mu \mathrm{g}$ of amphotericin B was slowly injected into the anterior vitreous cavity. Cultures from the scleral wound and the vitreous aspirate taken at the beginning of the operation yielded no growth.

Postoperatively intravenous amphotericin B (40$50 \mathrm{mg} /$ day) was started. The patient's serum creatinine was monitored and the dosage of amphotericin B adjusted. Oral prednisone $(80 \mathrm{mg} /$ day $)$ was continued. During the following 10 days the patient remained free of pain, and the anterior chamber and vitreous reaction gradually subsided. Three weeks after the lensectomy the optic disc could be seen, and yellowish exudates were noticed in the macula. Choroidal effusions were also observed, and they remained unchanged despite systemic prednisone therapy. One week later a choroidal tap was performed without complication. Fluid aspirated from the vitreous cavity for culture showed no growth. After 34 days of intravenous amphotericin B therapy (total dosage $1560 \mathrm{mg}$ ) antifungal treatment was dis-

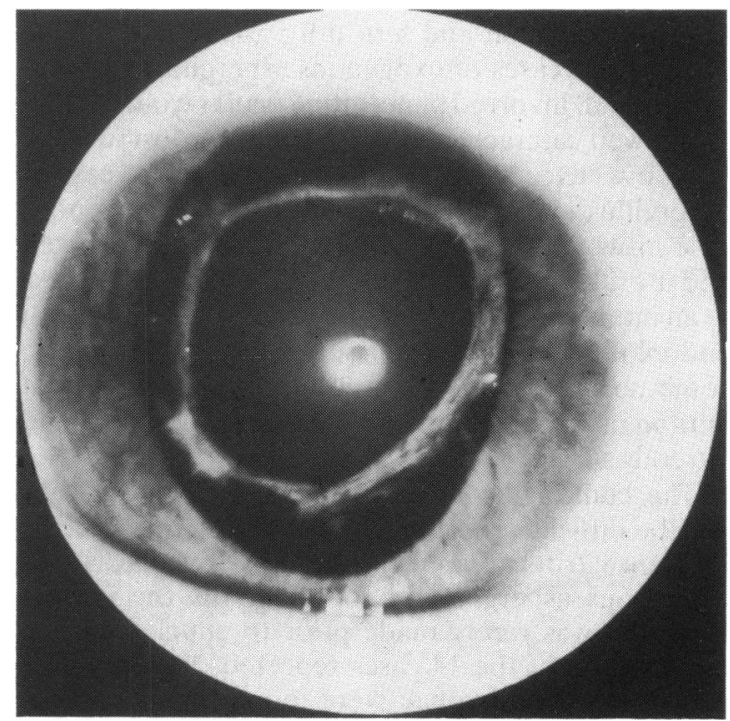

Fig. 2 External photograph of the right eye 3 months after the onset of endophthalmitis. The media were clear. Visual acuity was $6 / 18$. 


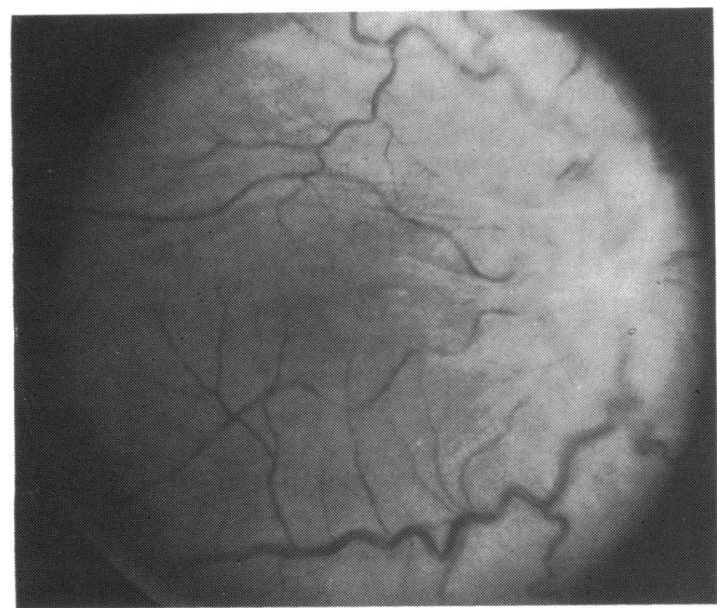

Fig. 3 Fundus photograph 3 months after the onset of endophthalmitis. A preretinal membrane was present in the macular area.

continued. The patient was discharged from hospital on the 35 th day.

Three months later visual acuity in the right eye, with refraction, was $6 / 18$. The media were clear (Fig. 2). A preretinal membrane in the macula prevented further visual improvement (Fig. 3).

\section{Discussion}

To our knowledge 24 cases of endogenous aspergillus endophthalmitis have been reported. Fifteen of those cases occurred in already ill people and patients with systemic infection and 5 in drug abusers. ${ }^{1-3}$ Of the 14 reported cases of exogenous aspergillus endophthalmitis 10 involved penetrating ocular wounds, and 4 followed cataract surgery. ${ }^{4}$ Vision was lost in all of these eyes except $2 .{ }^{13}$ Our patient is the third case of aspergillus endophthalmitis and the first exogenous case in which the infection was successfully treated and useful vision preserved. We attribute our success to an intensive approach in the management of fungal endophthalmitis characterised by rapid diagnosis, combined use of multiple intravitreous instillations of antifungal agent and intravenous therapy, and vitreous surgery.

The clinical diagnosis of fungal endophthalmitis may be difficult, since it can appear as a non-specific indolent vitritis or uveitis. ${ }^{56}$ In the reported cases of exogenous aspergillus endophthalmitis the correct diagnosis was rarely made prior to enucleation. In fact in most of the 14 cases reported ${ }^{4}$ the diagnosis was made when hyphae were found by histopathological examination of ocular tissue after enucleation. Positive cultures for the fungus obtained from the excised tissue further confirmed the diagnosis. In our case a diagnostic vitreous tap was performed as soon as endophthalmitis was considered a possibility, and a latent period of 16 days further heightened our suspicion of fungal involvement. Optimally, with penetrating injuries a wet preparation for fungi should be examined at the time of initial vitrectomy. When aspergillus was recovered from the culture, appropriate antifungal therapy was started immediately. This is the first exogenous case of aspergillus endophthalmitis in which the diagnosis was confirmed by culture before the eye succumbed to the infection.

Amphotericin $B$ is the antifungal agent of choice in the management of aspergillus infection. However, no case of aspergillus endophthalmitis has been successfully treated with intravenous amphotericin B alone because this compound does not penetrate well into the vitreous. ${ }^{7-9}$ Direct and slow intravitreous injection of amphotericin B is the only way to achieve intraocular therapeutic levels. Although one recent study showed that up to $10 \mu \mathrm{g}$ of this drug injected into the vitreous cavity of rabbit caused no damage. ${ }^{10}$ another study demonstrated the occurrence of localised retinal necrosis after a total dose of $1 \mu \mathrm{g} .{ }^{11}$ In reported cases of aspergillus, ${ }^{1}$ candida, ${ }^{12}$ and paecilomyces ${ }^{13}$ endophthalmitis in man total intravitreous doses of 5 to $50 \mu \mathrm{g}$ of amphotericin B have not caused serious retinal toxicity. Marked intraocular inflammation after intraocular amphotericin B injection could be caused by persistent fungal infection, the enzymes released from the dying fungi, the toxic effects of the drug, or by a combination of these. Therefore multiple vitreous taps for fungal culture would help monitor the effectiveness of the antifungal treatment when the clinical signs were difficult to interpret. When the risk of retinal toxicity precludes further intravitreous drug injection, or when the infection progresses despite such therapy, combined synergistic treatment with systemic amphotericin B and rifampicin or fluocystosine could be considered. ${ }^{14} 15$

We believe that vitrectomy is indicated when fungal endophthalmitis is suspected. In the acute stage vitrectomy can confirm the diagnosis with a vitreous biopsy for pathology as well as wet preparation and culture. ${ }^{16}$ Vitrectomy excises the bulk of the organism and inflammatory cells as it drains the vitreous abscess. When the gel is removed, the vitreous cavity is more permeable to large molecules, and the delivery and diffusion of antifungal agents are facilitated. Vitreous surgery prevents the long term complications of intraocular inflammation by minimising the development of vitreoretinal traction and subsequent retinal detachment. Retrolental and cyclitic membranes formed after severe intraocular inflammation not only obstruct the fundus view in the management of endophthalmitis but also contribute to ocular hypotony. Vitrectomy combined with lensec- 
tomy provides an approach to remove these membranes and to restore other structural alterations. However, intraocular surgical manipulations in an inflamed eye have potentially adverse effects. Surgical complications may occur, and the toxic effect of intravitreous antifungal drugs following vitrectomy is unknown.

A vigorous diagnostic and therapeutic approach in the management of fungal endophthalmitis has been successful in recently reported cases. ${ }^{1316}$ We believe the prognosis of this devastating intraocular infection can be improved with vitrectomy, multiple vitreous aspirates for culture, and systemic antifungal therapy combined with repeated intravitreous injections of an antifungal agent.

\section{References}

1 Doft BH, Clarkson JG, Rebell G, Forster RK. Endogenous aspergillus endophthalmitis in drug abusers. Arch Ophthalmol 1980; 98: 859-62.

2 Wilmarth SS, May DR, Roth AM, Cole RJ, Nolan S, Goldstein E. Aspergillus endophthalmitis in an intravenous drug user. Ann Ophthalmol 1983; 15: 470-6.

3 Michelson JB, Freedman SD, Boyden DG. Aspergillus endophthalmitis in a drug abuser. Ann Ophthalmol 1982; 14: 1051-4.

4 Francois J, Rysselaere M. Oculomycoses. Springfield: Thomas, 1972: 77-9, 172 .
5 Snip RC, Michels RG. Pars plana vitrectomy in the management of endogenous candida endophthalmitis. Am J Ophthalmol 1976; 82: 699-704.

6 Stone RD, Irvine AR, O'Connor GR. Candida endophthalmitis: report of an unusual case with isolation of the etiologic agent by vitreous biopsy. Ann Ophthalmol 1975; 7: 757-62.

7 Naidoff MA, Green WR. Endogenous aspergillus endophthalmitis occurring after kidney transplant. Am J Ophthalmol 1975; 79: 502-9.

8 Green WR, Bennett JE, Goos RD. Ocular penetration of amphotericin B. A report of laboratory studies and a case report of postsurgical cephalosporium endophthalmitis. Arch Ophthalmol 1965; 73: 769-75.

9 Fisher JF, Taylor AT, Clark J, Rao R. Penetration of amphotericin B into the human eye. J Infect Dis 1983; 147: 164.

10 Axelrod AJ, Peyman GA, Apple DJ. Toxicity of intravitreal injection of amphotericin B. Am J Ophthalmol 1973; 76: 578-83.

11 Souri EN, Green WR. Intravitreal amphotericin B toxicity. Am J Ophthalmol 1974; 78: 77-81.

12 Stern GA, Fetkenhour CL, O'Grady RB. Intravitreal amphotericin B treatment of candida endophthalmitis. Arch Ophthalmol 1977; 95: 89-93.

13 Miller GR, Rebell G, Magoon RC, Kulvin SM, Forster RK. Intravitreal antimycotic therapy and the cure of mycotic endophthalmitis caused by a Paecilomyces lilacinus contaminated pseudophakos. Ophthalmic Surg 1978; 9: 54-63.

14 Corrado ML, Cleri D, Fikrig SM, Phillips JC, Ahonkhai VI. Aspergillosis in chronic granulomatous disease: therapeutic considerations. Am J Dis Child 1980; 134: 1092-4.

15 Karam GH, Dismukes WE. Pulmonary mycoses: choosing the right drug. J Respir Dis 1983; 4: 93-103.

16 Chen CJ. Nocardia asteroides endophthalmitis. Ophthalmic Surg 1983; 14: 502-5. 